\title{
Comparative Analysis of Different Mobility Protocols for VHO based on MIH Standards
}

\author{
R. Tamijetchelvy \\ Asst. Professor, Dept. of ECE \\ PKIET, Karaikal \\ Pondicherry, India
}

\author{
C. Hepzibah \\ Dept. of ECE \\ Pondicherry Engg. College \\ Pondicherry, India
}

\author{
G. Sivaradje \\ Professor, Dept. of ECE \\ Pondicherry Engg. College \\ Pondicherry, India
}

\begin{abstract}
Mobile user service continuity across heterogeneous wireless access network is a challenging task for designing future generation wireless networks. Existing communication platforms may efficiently utilize heterogeneous architecture rather homogeneous solution for both non-real-time and realtime applications. Such seamless service continuity is obtained by proper vertical handover algorithms and mobility protocols across various layers to satisfy the user needs. Therefore, efficient standards are necessary for maintaining service quality in heterogeneous networks. One such standard is IEEE 802.21 MIH which provides media-independent framework and associated services to enable seamless handover between heterogeneous networks. In this paper different mobility management protocols such as PMIPv6, MIPv6, HMIPv6 and FMIPv6 are analyzed, implemented and compared for integrated WLAN / WiMAX networks in terms handover latency and throughput.
\end{abstract}

\section{General Terms}

Vertical Handover based on IEEE 802.21 Media Independent Handover Standards.

\section{Keywords}

IEEE 802.21 MIH, MIPv6, PMIPv6, HMIPv6, FMIPv6, WiMAX, Vertical Handover, WLAN.

\section{INTRODUCTION}

Existing mobile network architecture is homogeneous and centralized in nature, which is completely unsuitable for present mobile internetworking environment. Since the mobile user may roam anywhere and want to access any service at any-time. The quality of service, cost, security, power consumption and resource utilization plays a major role in heterogeneous environment. Therefore proper algorithms are needed to cope the mentioned requirements into account irrespective of different access technologies. More networks are available (WLAN, WiMAX, UMTS etc.) to satisfy the user needs and support best QoS guarantee. Handover across these technologies should be seamless and avoids service termination, especially for delay sensitive applications. Therefore efficient handover algorithm is necessary for seamless service continuity. In addition existing mobility management protocols should need to be integrated or better communication. The ongoing communication should be seamlessly transferred from old point of attachment to new point of attachment during handover process. Lots of effects have been carried out for vertical handover decision in heterogeneous solution. Service quality should be well maintained during and after handover process.

The recent emerging approach to deals vertical handover procedures is by considering the IEEE 802.21 MediaIndependent Handover (MIH) Services to enhance handover operation when the users moves between different access technology. The IEEE $802.21 \mathrm{MIH}$ working group provides sufficient solution [1] for efficient handover signaling across heterogeneous environment. The implementation module of MIH standard is introduced in [2] with stream control transmission protocol for VoIP applications. SIP concept is introduced in [3, 4] for low level mobility support. Later [5, 6] proposed handover decision algorithm for minimizing handover failure and unnecessary probability by prediction of handover in advance. The PMIPv6 based handover issues are discussed in [7] for real time video applications. The IEEE 802.21 MIH standard support seamless handover in heterogeneous architecture without service interruption as discussed in $[8,9]$. Later [10] introduced centralized structure for optimized handover signaling across wireless environments. The remaining of the chapters is organized as follows. The following chapter describes an outline of the IEEE 802.21 MIH standard, and the section after that provides an overview of different mobility management schemes. The Section IV defines the presented integrated architecture. The simulation results are presented in section $\mathrm{V}$ and finally conclude the challenges and future scope.

\section{IEEE 802.21 MIH STANDARD}

IEEE has developed a standard called as Media Independent Handovers (MIH) defined in IEEE 802.21. The IEEE 802.21 provides sufficient information for efficient handover optimization in heterogeneous environment. The proper handover signaling for necessity condition are clearly discussed irrespective of different wireless access technologies. It lies in layer L 2.5 protocol stack and provides intelligent decision. Each and every function are clearly illustrated and explained in the following sections. Therefore this novel standard should be adopted in the heterogeneous architecture to enhance fast and seamless connection when the mobile node changes its point of attachment. The protocol stack of MIH standards allows a framework for transparent continuity by group of handover enabling functions and Service Access Point (SAP).

The main heart of MIH is the MIH Function (MIHF) which lies in between MIH User (MIHU) and lower mobile node interface as in Fig. 1. It supports three important services Media Independent Event Service (MIES), Command Service (MICS) and Information Service (MIIS). The MIHU is adopted in network side and MIHF is in user side in order to report its current status to the upper higher layers.

$M I H F$ : the function of MIHF is to report the lower layer link information to upper MIHU layer through well-defined service access point (SAP). The MIH_LINK_SAP provides intelligent signaling exchange between the MIHF and lower device interface.

\subsection{MIH Services - An Overview}

The IEEE 802.21 MIH standard defines three important services for optimized handover across heterogeneous architecture namely: Event service, Command service and 
Information service. These services are considered as primary for initiating intelligent handover decision across heterogeneous environment and extremely well applicable for designing future generation networks.

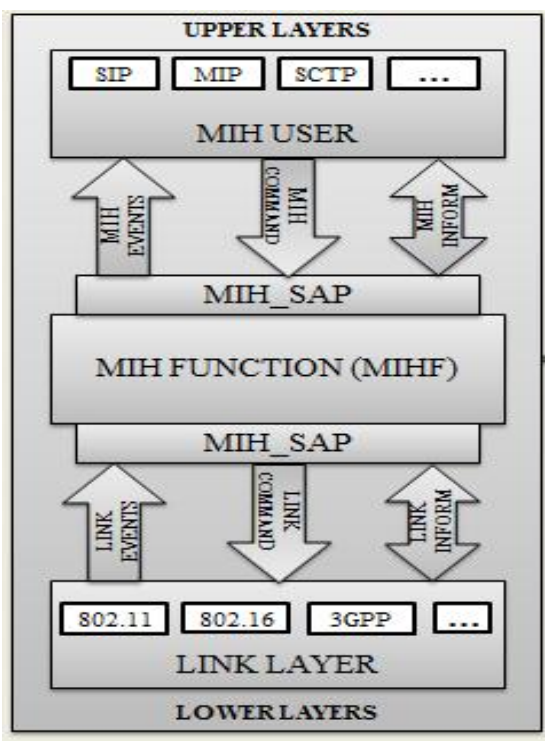

Fig 1: IEEE 802.21 MIH Standard

\section{A. Media Independent Event Services}

The Event service defines the changes in link characteristics such as link status, link condition and link quality etc. The MIES classified into two basics: link event and MIH event. Link Events are the event information's from the lower physical interface to the middle MIHF, while the MIH events start from MIHF and finally reaches to MIHU. Events are exchanged from peer entities through MIH function. Three main important events are triggered for lower layer to upper layer for efficient handover execution. They are Link_Up, Link_Down and Link_Going_Down triggers.

\section{B. Media Independent Command Services}

The command service is used to cope and control the link state behavior. The commands are sent from the upper MIHU to lower link layer. The MIHU use command services to obtain the link status for the multi-mode device to utilize heterogeneous networks efficiently. For example, once it receives the link going down event, the MIHU will initiate the handover necessity command to the link layers. The network also initiates handover decision with MIHU for proper load balancing during handovers operations.

\section{c. Media Independent Information Service}

The information service provides necessary information about the neighbor network over the geographical area by querying and responses. The MIIS collects the information about adjacent network during network deployment phases. The MIIS also provides static and dynamic information of neighbor node for excellent and optimized handover operation.

\subsection{MIH Protocol Header}

The MIH header defines the protocol rules for communicating any access technologies which follows services between peer end to end MIHF entities. The protocol header consists of eight octets as depicted in Fig 2. The header format deeply describes the length in each field [11]. The version indicates the MIH protocol version number. Two acknowledgment fields for request and response of information are used. The
UIR is the unauthenticated information request which uses minimum length. If UIR flag is set, fails to provide registered information.

\begin{tabular}{|c|c|c|c|c|c|c|c|c|}
\hline \multirow[b]{2}{*}{$\begin{array}{l}\text { Version } \\
(4)\end{array}$} & \multirow{2}{*}{$\begin{array}{l}\text { ACK } \\
\text { Request } \\
\text { (1) }\end{array}$} & \multirow{2}{*}{$\begin{array}{c}\text { ACK } \\
\text { Response } \\
\text { (1) }\end{array}$} & \multirow[b]{2}{*}{$\begin{array}{l}\text { UIR } \\
\text { (1) }\end{array}$} & \multirow[b]{2}{*}{$\begin{array}{ll}\text { M } & \text { FN } \\
(1) & (7)\end{array}$} & \multirow[b]{2}{*}{$\begin{array}{l}\text { RSVD } \\
\text { (1) }\end{array}$} & \multicolumn{3}{|c|}{ MIH Message ID(16) } \\
\hline & & & & & & $\underset{(4)}{\operatorname{SID}}$ & $\begin{array}{l}\text { Opcode } \\
\text { (2) }\end{array}$ & $\begin{array}{l}\text { AID } \\
(10)\end{array}$ \\
\hline $\begin{array}{c}\text { RSVD } \\
(4)\end{array}$ & \multicolumn{5}{|c|}{ TransactionID (12) } & \multicolumn{3}{|c|}{$\begin{array}{c}\text { Variable Load Length } \\
\text { (10) }\end{array}$} \\
\hline
\end{tabular}

Fig 2: MIH protocol header

\section{OVERVIEW OF THE MOBILITY MANAGEMENT FUNCTIONS}

The mobility function is an important issue in the heterogeneous architecture. The mobility functions are handled smoothly by mobility protocols across various layers (MIP, SIP, and SCTP etc.). At the link layer, mobility concerns a change in the base station. At that time, the ongoing session need to be enhanced to candidate point of attachment independent of access technologies. The static and dynamic information of the neighbor network such as RSS (received signal strength), resource query are essential during handover operation. Hence IEEE 802.21 MIIS server provides information about adjacent network for making seamless handover across heterogeneous networks. At the network layer, mobility management is the current point of attachment changes. It adopts routing and mapping based mechanism for intelligent mobility management. In routing based scheme, the IP address of host is unchanged throughout the communication process. Whereas in mapping based solution, the IP prefix changes when the mobile node changes its current point of attachment. Mapping-based network-layer mobility solutions include Mobile IP (MIP), Proxy Mobile IP (PMIP), Fast handover for MIP (FMIP) and Hierarchical Mobile IP (HMIP).

At the transport layer, mobility management is about end to end delay between peer entities. Mobility solutions handle how to migrate TCP connections when the IP address of a TCP end-node changes. In application layer, session should be seamlessly carried out without service degradation particularly for delay sensitive applications.

\subsection{Network Layer Solution}

The network layer provides a solution to mobility management for any type of traffic classes. Among all the solutions, Mobile IP (MIP) is the most well-known networklayer mobility solution. Mobile IP protocol allows nodes to remain reachable while moving around in the IP internet. Mobile nodes are always identified by their unique address, irrespective of their current address to the internet and also it is also associated with a Care-of-Address $(\mathrm{CoA})$ when it is located far from the serving home domain, which provides sufficient information about the MN current point of attachment. The IP packets to the MN home prefix are transparently routed to its care-of-address. In homogenous and heterogeneous media, MIP supports bidirectional tunneling and route optimization for location management. They both use binding updates to the Home Agent (HA) and Corresponding Nodes (in case of route optimization) whenever they change their point of attachment to the internet. Handoff management is done in the similar way, the corresponding node creates a connection with the Home Agent and in the case of handoff the Mobile Nodes open up a new connection with Home Agent. This makes handoff transparent from the corresponding node. This behavior of movement transparency of the mobile node becomes void in the case of route optimization. 


\subsection{Mobility Management Protocols}

The next generation networks are heterogeneous in nature to support seamless connection is a challenging task. Various groups working toward centralized architecture for efficient handover optimization. The mobility management protocols across various layers such as SIP (Session Initiation Protocol), SCTP (Stream Control Transmission Protocol), Mobile IPv6 (MIPv6), Fast Handovers for Mobile IPv6 (FMIPv6), Hierarchical Mobile IPv6 (HMIPv6), Proxy Mobile IPv6 (PMIPv6) and Fast Handovers for PMIPv6 (Predictive FPMIPv6, and Reactive FPMIPv6) etc. to be properly adopted for intelligent handover optimization.

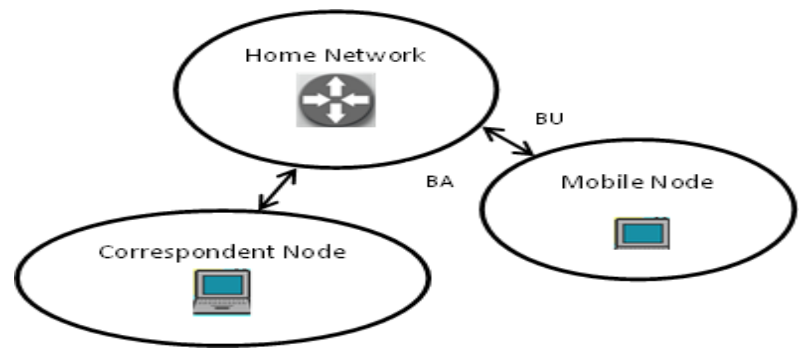

Fig 3: MIPv6 operation

\section{A. MIPv6}

Mobile IPv6 was designed to allow nodes to be reachable and maintain ongoing connections while changing their location within the topology. Mobile IPv6 uses a stable IP address, assigned to mobile nodes (home address). The home address is used for two reasons: first, to allow a MN to be reachable by having a stable entry in the DNS, and second, to hide the IP prefix mobility from upper layers. Fig 3 depicts the signaling flow of MIPv6 protocol. Tunneling is required to ensure the transparency of the service provided by the home agent.

\section{B. PMIPv6}

MIPv6 is a host based mobility protocol in which mobility signaling is handled by mobile nodes. The handover signaling information is triangular based routing and increases signaling overhead. This results in maximum handover delay. Hence network based PMIPv6 solution is introduced to for efficient handover. The MN does not involve in signaling information. The mobile node binding updates are done in Localized Mobility Anchor (LMA) which is centralized. Fig.4 illustrates the signaling flow of PMIPv6 protocol.

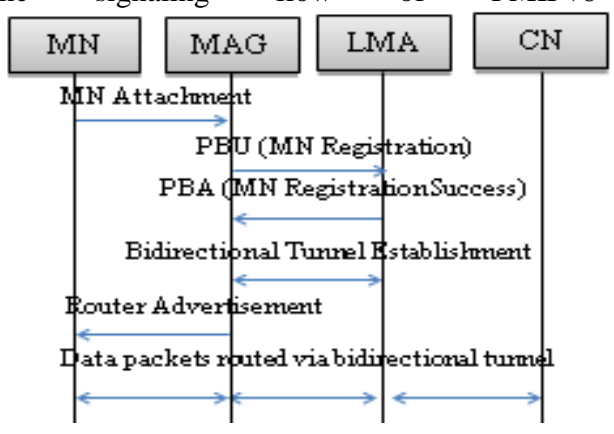

The elements of PMIPv6 are mobile access gateway (MAG) and local mobility anchor (LMA). The MAG typically runs on the access router (AR). The main role of the MAG is to detect the MN's mobility and initiates mobility signaling MA using

Proxy Binding Update and Acknowledgement Messages

(PBU and PBA) without involvement of MN. Later it establishes bidirectional tunnel between new MAG and LMA to carry-out the ongoing session. The main role of the LMA is to maintain the global binding cache entry of all registered mobile nodes. Finally the data packets are routed via bidirectional tunnel.

\section{HMIPv6}

HMIP is an extension to Mobile IP for micro-mobility Management. It is used to reduce the amount of signaling between the Mobile Node, its Correspondent Nodes, and its Home Agent to improve the handover latency. Fig 5 shows the architecture of HMIPv6. HMIPv6 involves three phases:

- MAP Discovery

- MAP Registration

- $\quad$ Packet Forwarding

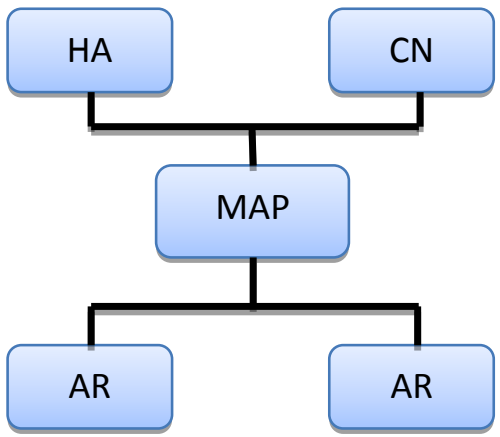

Fig 5: HMIPv6 Architecture

\section{FMIPv6}

Fast handovers for Mobile IPv6 protocol enables the mobile user to communicate to new point of attachment efficiently. It takes less time to compute handover between different access technologies and thus reduces service disruption.

The FMIPv6 provides buffering mechanism to receive data packets during handover. The handover signaling information such as handover initiate and acknowledgement are exchanged in a secure way by FBU message from MN. The buffering mechanism helps to retrieve the data packets and thus reduces packet dropping ratio. There are two types for fast handover: Predictive fast handover and Reactive fast

\section{IMPLEMENTATION OF ROUTING PROTOCOLS IN HETEROGENEOUS NETWORK}

Nowadays there are many mobile devices which do interoperability between different networks due to greater development in the field of software radio technology. The IP based infrastructure is the key part of next generation mobile systems which allows an efficient, cost-effective and seamless interworking between the overlay networks.

\subsection{Integrated WLAN / WiMAX Architecture}

By integrating the promising networks like WLAN and WiMAX will leads to several benefits of load balancing, extension of coverage area, improved Quality of Service (QoS), improved security features etc, which is the combined advantage of these three networks. On integrating the features and advantages of the three networks can utilize optimized handover.

\section{A. IEEE 802. 11 (WLAN)}

Mobile IPv6 IEEE 802.11 provides the standard for wireless connectivity for both fixed and portable devices that lies inside the local area. The primary services of the Wireless Local Area Network(WLAN) is to provide MAC service data 
units called Medium Access Protocol Data Units (MSPDU) between the Logical Link Control (LLC) layer. There are many highlighted features of WLAN as high bandwidth security and power management. The 802.11 standard allows stations to roam among set of APs connected to distributed systems. WLAN data functionality is provides by Physical Medium Dependent (PMD) responsible for modulation, Physical Layer Convergence Protocol (PLCP) and Medium Access Control (MAC) sublayer for security, packet ordering etc.

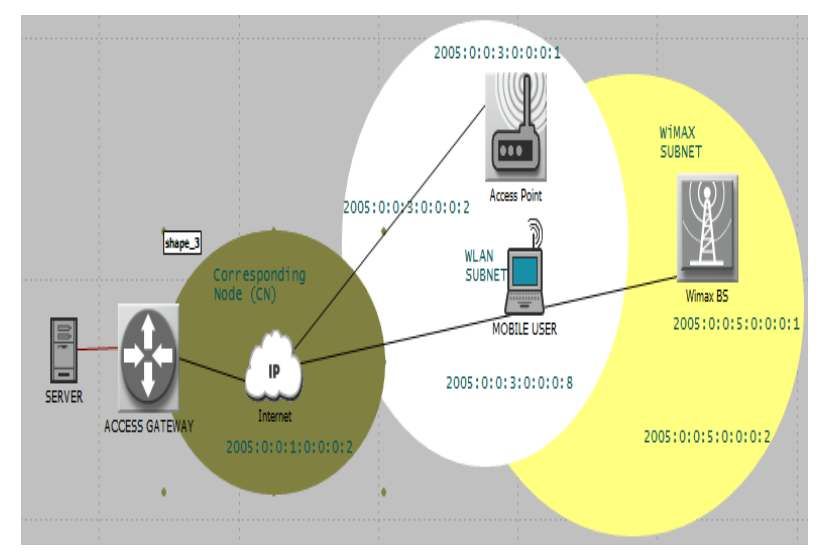

Fig 6: Implementation of MIPv6

\section{B. IEEE 802. 16 (WiMAX)}

Mobile IPv6 WiMAX is the Worldwide Interoperability for Microwave Access which is designed to provide the data rates of 30 to $50 \mathrm{Mbps}$ with the 2011 update providing up to 1 Gbits/s for fixed stations. The name "WiMAX" was created by the WiMAX Forum, which was formed in June 2001 to promote conformity and interoperability of the standard. WiMAX supports point to point and mesh mode. It uses MIMO in physical layer. Both the transmitter and receiver use multiple antennas in order to improve coverage, installation, bandwidth efficiency and performance.

\subsection{MIPv6 in WLAN / WiMAX Network}

Initially the mobile node is in WLAN coverage and exchanges their data with the corresponding node through IP connectivity. WLAN access point is connected with MIH user and mobile node is connected with MIH function. MIHF continuously measures the link quality and gets its status through MIES command, continuously measures the link quality and gets its status through MIES command. If the link going down event is triggered MIHU in WLAN accessing point initiates the need for handoff. Then the MIHU in WLAN accessing point discovers candidate network from the information server, then the server immediately informs to MIHU with its already scanned results i.e MIIS collects the information about the candidate networks with query and response. Fig 6 shows the implementation of MIPv6 protocol in WLAN-WiMAX wireless networks.

\subsection{PMIPv6 in WLAN / WiMAX Network}

A network layer PMIPv6 solution for handover optimality is achieved by centralized LMA and MAG access gateway. The LMA performs global binding for all registered mobile nodes. All the handover signaling information is passed via LMA and MAG.

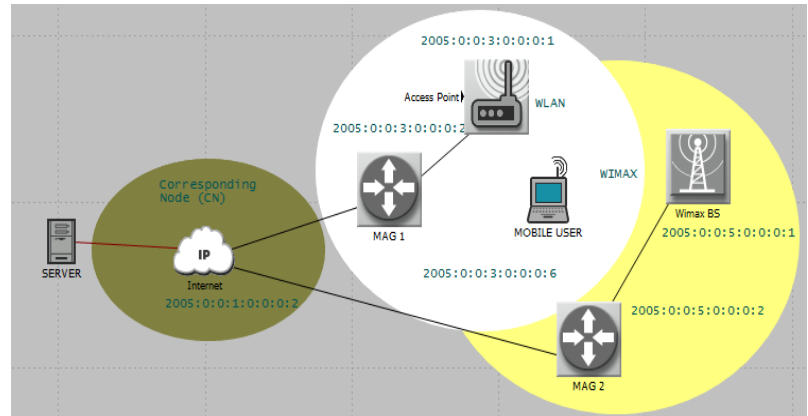

Fig 7: Implementation of PMIPv6

\subsection{HMIPv6 in WLAN / WiMAX Network}

HMIPv6 is used for micro mobility. The coverage area is very less when compared to other protocols as shown in Fig. 8. It uses previous access router and new access router to which the $\mathrm{MN}$ is connected.

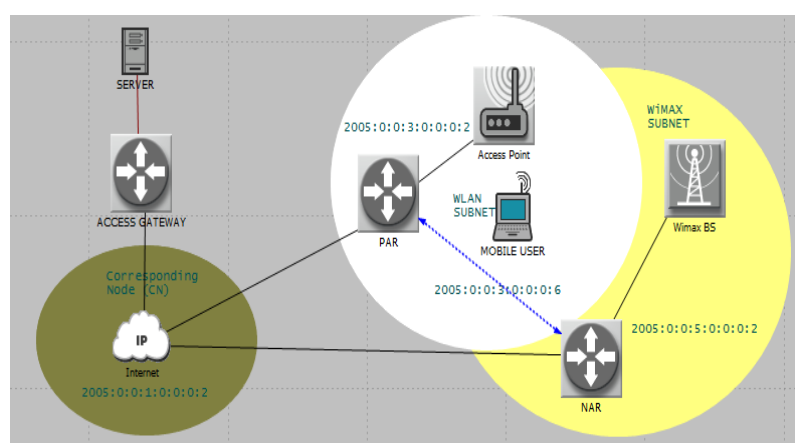

Fig 8: Implementation of HMIPv6

\subsection{FMIPv6 IN WLAN/WiMAX}

In Predictive Fast Handover mechanism, MN is able to send an FBU when it is attached to the PAR, which then establishes forwarding for its traffic. Reactive Fast Handover is the fast handover in which an MN is able to send the FBU only after attaching to the NAR It is shown in Fig. 9.

\section{SIMULATION RESULTS}

This section analyses different mobility protocols across two heterogeneous environments. The simulation done in OPNET modeler 14.5 software with the integration of two networks WLAN / WiMAX. The architecture consists of multi interface mobile node $(\mathrm{MN})$, correspondent node $(\mathrm{CN})$, application server with backbone IP connectivity. The results of different mobility management scenarios are given below.

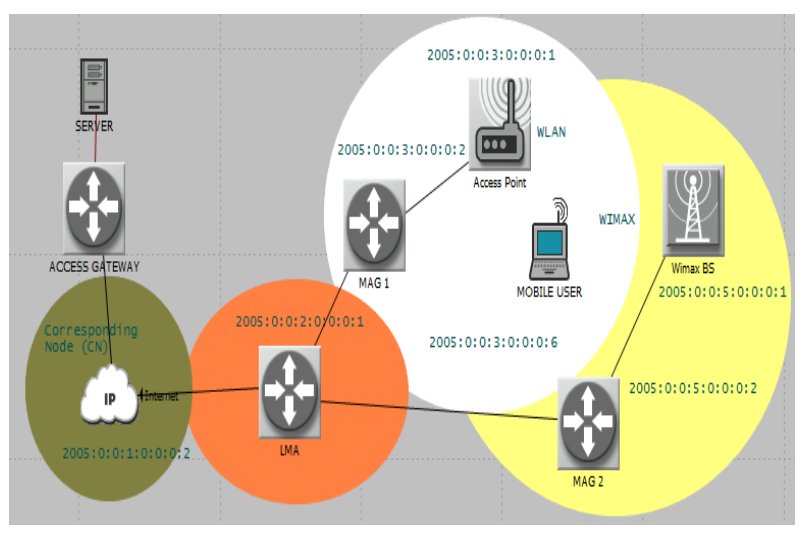

Fig 9: Implementation of FMIPv6 


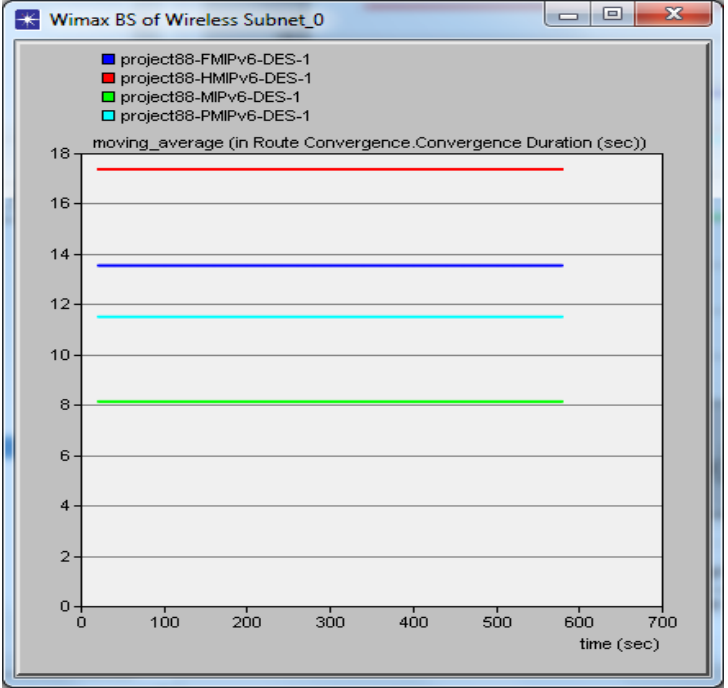

Fig 10: Route convergence duration

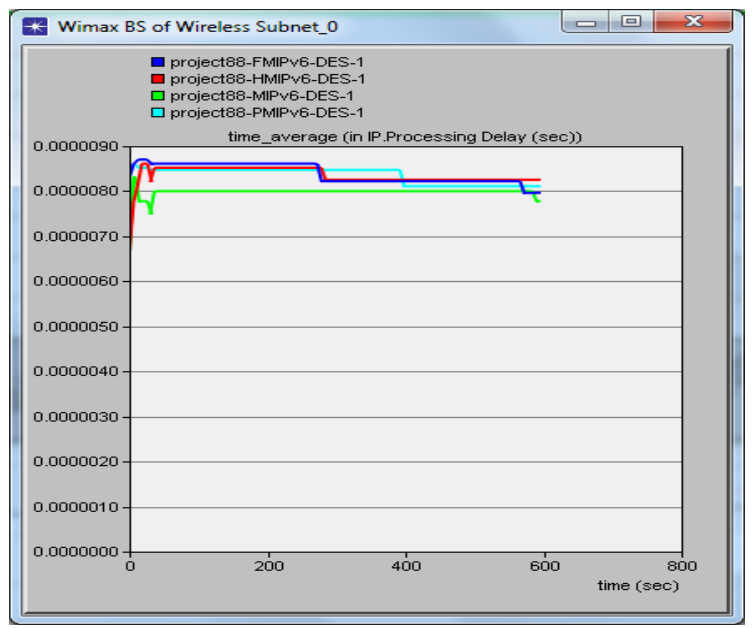

Fig 11: Processing Delay

From fig. 10, it is clear that the fastest routing convergence can be obtained by using MIPv6 protocol which is about 8 sec. Fig. 11 shows the processing delay of the integrated networks when applying these mobility protocols. The delay is less while using PMIPv6 protocol.

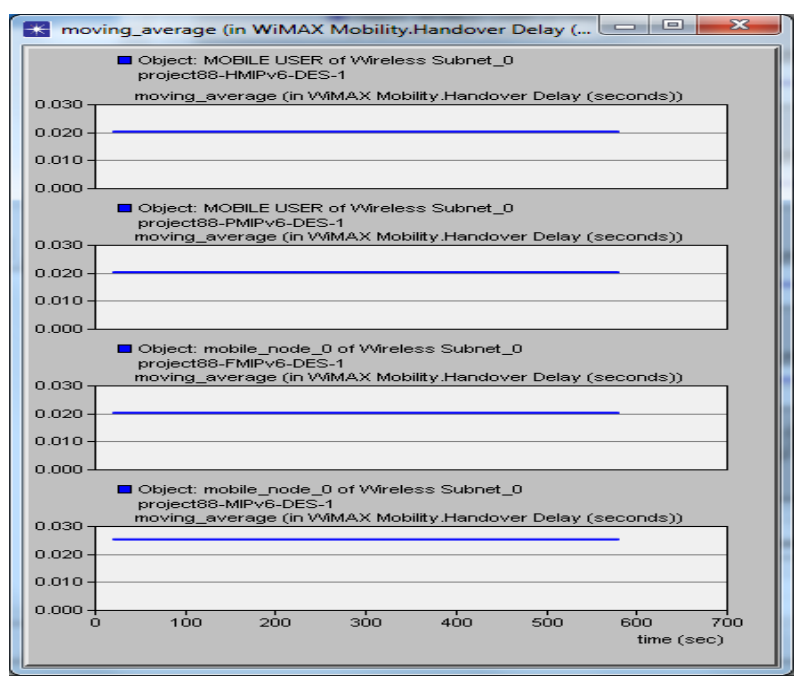

Fig 14: Handover Delay
Table 1 Comparison between mobility protocols

\begin{tabular}{|c|c|c|c|c|}
\hline Parameters & FMIPv6 & HMIPv6 & MIPv6 & PMIPv6 \\
\hline $\begin{array}{c}\text { Route } \\
\text { convergence } \\
\text { Duration (sec) }\end{array}$ & 13.90 & 17.90 & 8.10 & 11.85 \\
\hline $\begin{array}{c}\text { Processing } \\
\text { Delay( } \mu \text { s) }\end{array}$ & 8.8 & 8.5 & 8.7 & 8.4 \\
\hline $\begin{array}{c}\text { Total channel } \\
\text { power (dBm) }\end{array}$ & 8 & 8.5 & 8 & 8.5 \\
\hline $\begin{array}{c}\text { Throughput } \\
\text { (packets/sec) }\end{array}$ & 0.11 & 0.08 & 0.02 & 0.17 \\
\hline $\begin{array}{c}\text { WiMAX delay } \\
(\mathrm{sec})\end{array}$ & 0.0025 & 0.0055 & 0.0055 & 0.0035 \\
\hline $\begin{array}{c}\text { WiMAX load } \\
\text { (packets/sec) }\end{array}$ & 0.17 & 0.12 & 0.08 & 0.13 \\
\hline
\end{tabular}

\section{CONCLUSION}

The integrated WLAN / WiMAX architecture proves to provide an efficient handover by using IEEE 802.21 Media Independent Handover with different mobility protocols at network layer. This paper compares analyses the performance of different mobility protocols for integrated WLAN and WiMAX networks. The results reveal that the PMIPv6 protocol outperforms the other protocols in terms of handover latency, packet loss ratio and throughput which are extremely well applicable for designing next generation networks. It is proved that PMIPv6 protocol is well suited for both delay tolerant and delay sensitive applications. Future work aims to provide distributed mobility management solution in heterogeneous environments rather centralized environment and analyze its mobility behavior with existing protocols suitability.

\section{REFERENCES}

[1] IEEE 802.21 WG "IEEE Standard for Local and Metropolitan Area Networks Part 21: Media Independent Handover Services," IEEE Std. 802.21-2008, Jan. 2009.

[2] Tein-Yaw Chung,Yung-Mu Chen, Pu-Chen Mao, ChenKuan Tsai, Sheng-Wen Lai, Chun-Po Chen, "The Design and Implementation of IEEE 802.21 and its Application on Wireless VoIP“, IEEE 978-1-4244-2519-8, 2010.

[3] Q. Wang and M. A. Abu-Rgheff, "Integrated Mobile IP and SIP Approach for Advanced Location Management", IEEE 3G 2003, London, U.K, June 2003.

[4] Wei wu, Alcatel, Nilanjan Banerjee, Kalyanbasu and Sajal k. Das,"SIP-based vertical handoff between WWANs and WLANs", IEEE Wireless Communications, June 2005.

[5] S. Mohanty and I. F. Akyildiz," A Cross-layer (layer 2 +3) Handover Management Protocol for NextGeneration Wireless Systems", IEEE Transactions on Mobile Computing, 5:1347-1360, October 2006.

[6] Elaheh Arabmakki, Sherif Rashad and Sadeta Krijestorac,"An Algorithm for Optimizing Vertical 
Handoff between WLAN and Cellular Networks", Cyber journals: Multidisciplinary Journals in science and Technology, (JSAT), pp.17-22, 2011.

[7] Md. Mahedi Hassan, Poo Kuan Hoong, Seamless Handover integrated solution for Video Transmission over Proxy Mobile IPv6 in a micro mobility domain, Journal of Network and Computer Applications, 36 (2013), pp. 66-76.

[8] José Jailton, Tássio Carvalho, Warley Valente, Carlos Natalino, and Renato Francês, "A Quality of Experience Handover Architecture for Heterogeneous Mobile Wireless Multimedia Networks,"IEEE Communications Magazine, June 2013.
[9] Stenio Fernandes, "Vertical Mobility Management Architectures in Wireless Networks: A Comprehensive Survey and Future Directions," IEEE Communications Surveys \& Tutorials, Vol. 14, No. 1, First Quarter 2012.

[10] Ian F. Akyildiz, Jiang Xie and Shantidev Mohanty, "Survey of Mobility Management in Next-Generation All-IP-Based Wireless Systems," IEEE Wireless Communications, vol.16, pp.16-28, 2004.

[11] Abraham George, Anup Kumar et.al "Protocols for mobility management in heterogeneous multi-hop wireless networks," Elsevier journal on Pervasive and Mobile Computing, vol.4, pp. 92-116, 2008. 